\title{
Florística del bosque mesófilo de montaña de Monte Grande, Lolotla, Hidalgo, México
}

\author{
Floristics of the cloud forest of Monte Grande, Lolotla, Hidalgo, Mexico
}

\author{
Armando Ponce-Vargas, Isolda Luna-Vega*, Othón Alcántara-Ayala y Carlos A. Ruiz-Jiménez \\ Departamento de Biología Evolutiva, Facultad de Ciencias, Universidad Nacional Autónoma de México, Apartado postal 70-399, México 04510, D.F., \\ México \\ *Correspondencia: ilv@hp.fciencias.unam.mx
}

Resumen. Se presenta un estudio florístico del bosque mesófilo de montaña del municipio de Lolotla, localizado al noreste del estado de Hidalgo. El área está enclavada en la Sierra Madre Oriental, dentro de la región de la Huasteca hidalguense. Se ofrece un listado florístico de las plantas vasculares, compuesto por 103 familias, 260 géneros, 359 especies y 11 taxa subespecíficos. En este bosque hay 11 especies que están en alguna categoría de riesgo dentro de la Norma Oficial Mexicana NOM-050-ECOL-2001 y se sugiere el estudio de dos más para su posible inclusión.

Palabras clave: bosque mesófilo de montaña, florística, diversidad florística, Monte Grande, Lolotla, Hidalgo, México.

Abstract. A floristic inventory of the cloud forest of the municipality of Lolotla, in the state of Hidalgo was undertaken; this area is located in the Sierra Madre Oriental and is part of the Huasteca Hidalguense region. A floristic checklist of vascular plants composed by 103 families, 260 genera, 359 species and 11 subspecific taxa was obtained. Eleven species represented in this forest are included in the official Mexican document named Norma Oficial Mexicana NOM050-ECOL-2001, as species in some risk category and we suggest the study of two additional species for their possible inclusion.

Key words: cloud forest, floristics, floristic diversity, Monte Grande, Lolotla, Hidalgo, Mexico.

\section{Introducción}

Los bosques mesófilos de montaña mexicanos (Rzedowski, 1978) representan un tipo de vegetación intermedia entre la vegetación tropical y la templada, a diferencia de los de otras partes del mundo (Meave et al., 1992; Challenger, 1998). Se definen por la mezcla de elementos de muy diversas afinidades (Miranda y Sharp, 1950; Rzedowski, 1978) y se considera que tienen una composición biótica híbrida. En el país presentan una distribución geográfica en forma de archipiélago, donde cada isla tiene una composición biótica característica, dependiendo de la altitud, latitud, humedad, clima y suelo propios de cada lugar. Una definición más completa de este tipo de vegetación en México puede ser revisada en Rzedowski (1978) y en Challenger (1998), entre otros.

Hidalgo ocupa el tercer lugar en el país en cuanto a superficie de bosque mesófilo de montaña (Ortega y Castillo, 1996).

Recibido: 04 noviembre 2005; aceptado: 23 junio 2006
Entre los estudios florísticos y de vegetación más importantes sobre el bosque mesófilo en el estado de Hidalgo se encuentran los de Paray (1946, 1949), quien realizó excursiones botánicas en los municipios de San Bartolo Tutotepec, los cerros de Agua Blanca, Cumbre de Muridores y Tenango de Doria, donde registró algunas especies propias de estas zonas. Miranda y Sharp (1950) recorrieron los municipios de Zacualtipán, Chapulhuacán y Tenango de Doria, donde llevaron a cabo descripciones florísticas y fisonómicas. Puig (1976) realizó trabajos más completos en los bosques de los municipios de Tenango de Doria, Tlahuelompa y Tlanchinol, describiendo aspectos relacionados con el suelo, el clima, la fisonomía y estructura de los bosques. Más recientemente, Luna et al. (1994) publicaron trabajos más extensos sobre la composición florística del bosque de Tlanchinol; Alcántara y Luna (1997) describen la del bosque de Tenango de Doria; Mayorga et al. (1998), del bosque de Molocotlán, Molango-Xochicoatlán, y Alcántara y Luna (2001) realizaron trabajos florísticos en los municipios de Eloxochitlán y Tlahuelompa; por último, Escutia (2004) 


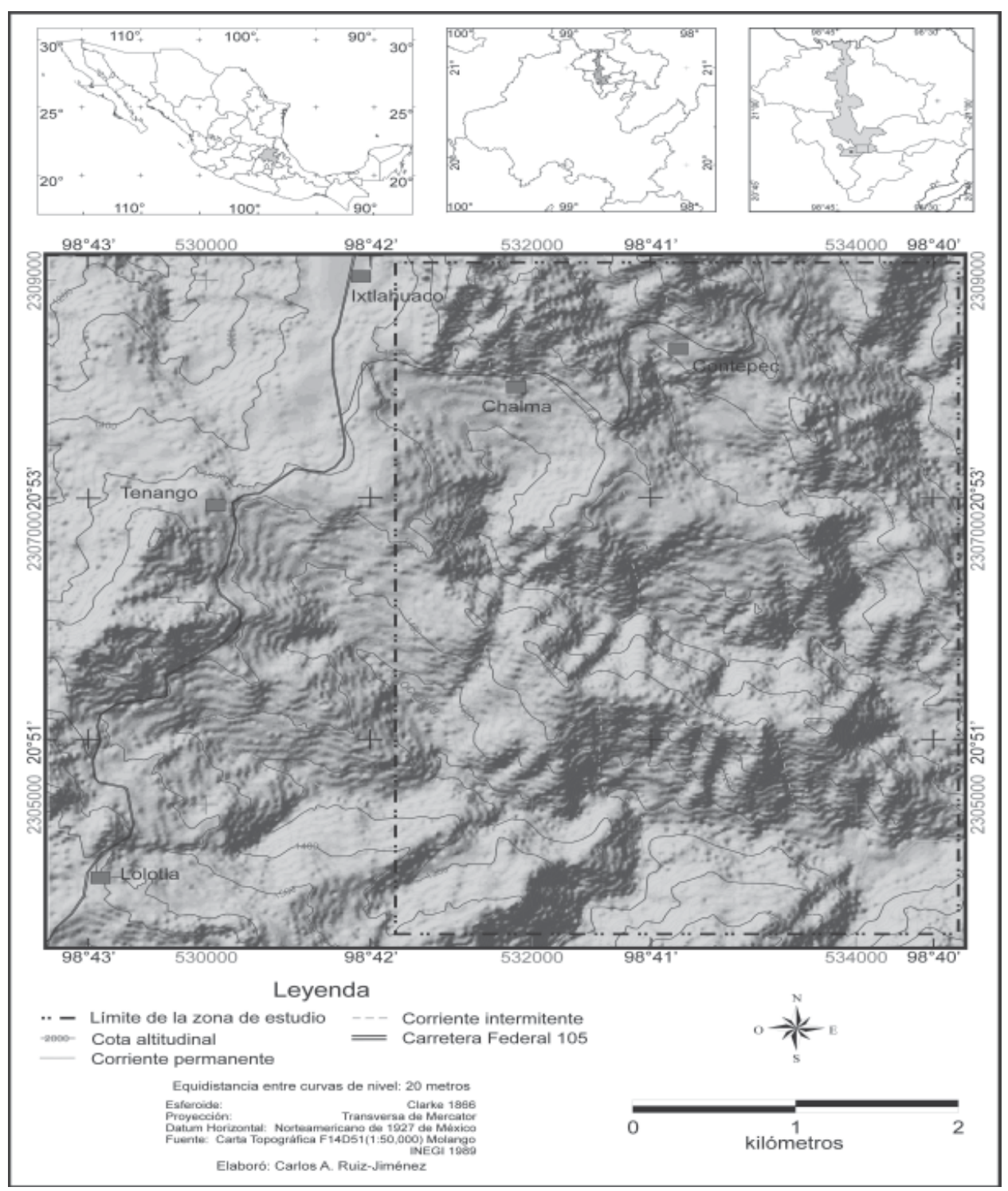

Figura 1. Mapa del área de estudio. (A) estado de Hidalgo; (B) municipio de Lolotla; (C) ubicación del área de estudio; (D) área de estudio.

describe la estructura de la vegetación en la región de Monte Grande de Lolotla.

La finalidad del presente trabajo es contribuir al conocimiento florístico de los bosques montanos de México en general y de la Sierra Madre Oriental y el estado de Hidalgo en particular. Este bosque está ubicado en la parte norte de la Región Terrestre Prioritaria 102, llamada Bosques mesófilos de la Sierra Madre Oriental (Conabio, 2000), por lo que es una de las zonas donde deben llevarse a cabo estudios de biodiversidad. El bosque del área de Lolotla constituye uno de los últimos fragmentos de bosque mesófilo de montaña, que aunque a primera vista parece similar a los manchones cercanos, posee una composición florística particular y propia.

\section{Materiales y métodos}

Área de estudio. El municipio de Lolotla se encuentra al noreste del estado de Hidalgo, entre los $20^{\circ} 53^{\prime} 20^{\prime \prime}$ ' y $20^{\circ}$ 50' $20^{\prime}$ ' N y $98^{\circ} 42^{\prime} 08^{\prime}$ ' y $98^{\circ} 40^{\prime} 12^{\prime}$ ' O. Limita al norte con el estado de San Luis Potosí, al sur con los municipios de Molango de Escamilla y Xochicoatlán, al este con los municipios de Calnali, Tlanchinol, San Felipe Orizatlán y 
Huazalingo, y al oeste con los municipios de Tepehuacán de Guerrero y Molango de Escamilla. La zona de estudio se encuentra entre los 1050 y 1580 m de altitud (Fig. 1). La superficie muestreada fue aproximadamente de 1022 hectáreas.

Para acceder a la zona de estudio desde la Ciudad de México, se debe tomar la carretera federal 85, MéxicoPachuca; posteriormente, la desviación que lleva hacia la carretera federal 105 Pachuca-Tampico. A la altura del km 143 se encuentran los terrenos comunales de Chalma y Tenango, de los cuales forma parte el bosque mesófilo de montaña de Monte Grande de Lolotla (SCT, 1987).

Fisiográficamente, la zona de estudio corresponde a la región norte del estado de Hidalgo, representada por la subprovincia del Carso Huasteco dentro de la provincia geológica y fisiográfica de la Sierra Madre Oriental, originada a fines del Mesozoico y principios del Cenozoico. Al noreste de la sierra se encuentra la región de la Huasteca, formada por lomeríos y sierras húmedas con buen drenaje (INEGI, 1992).

La zona de estudio se encuentra dentro de la región hidrológica del río Pánuco (RH26). Entre las corrientes permanentes de esta región se encuentra el río Atlapezco, que desemboca en el río Moctezuma (SPP, 1983b). Asimismo, dentro de los límites de Lolotla corre el río Xocontla en dirección noreste (Fig. 1).

Se presentan dos estratos geológicos; uno corresponde al Triásico, con asociaciones de rocas sedimentarias de areniscas y conglomerados; el otro, al Jurásico superior, con rocas sedimentarias de calizas y lutitas (SPP, 1983a).

Los suelos son arcillosos y se derivan de rocas sedimentarias mesozoicas, ricos en materia orgánica y nutrientes (INEGI, 1995), de tipo regosol éutrico (INEGI, 1999).

El área de estudio carece de estación meteorológica propia. La más cercana es la de Tlanchinol, con el mismo tipo de vegetación. Presenta un clima semicálido húmedo, (A) $\mathrm{Cb}(\mathrm{fm})\left(\mathrm{i}^{\prime}\right) \mathrm{gw}$ ", templado, con lluvias en verano, verano fresco y largo, temperatura media del mes más frío entre 3 y $18^{\circ} \mathrm{C}$, poca oscilación térmica y marcha de temperatura tipo ganges (SP, 1970; Conabio-Estadigrafía, 1997; García, 2004). La temperatura media anual en el municipio es de $18^{\circ} \mathrm{C}$.

Procedimiento. Para llevar a cabo este trabajo se realizó una visita prospectiva a la zona de estudio, además de hacer un análisis cartográfico [mapa de carreteras (SCT, 1987), cartas topográficas (INEGI, 1989) y fotografías aéreas (CETENAL, 1976; INEGI, 1995)], por medio de los cuales se eligieron los sitios de recolecta, procurando aquellos con vegetación mejor conservada.

El trabajo de campo se realizó de octubre del 2000 a octubre del 2002, en todas las estaciones y en casi todos los meses del año, con salidas que tuvieron duración de 4 a 5 días por mes (aproximadamente 90 días). Se recolectaron más de 1000 especímenes botánicos (fanerógamas y pteridofitas) en estado fértil (floración y/o fructificación) y se hicieron observaciones particulares referentes a la planta o al lugar donde fue recolectada la misma.

El material colectado se herborizó de acuerdo con los procedimientos botánicos convencionales (Lot y Chiang, 1986) y posteriormente se identificaron las especies, con la ayuda de especialistas para algunas familias. El ordenamiento de las familias se llevó a cabo de acuerdo con el sistema de Engler y Diels (1936) en el caso de angiospermas y gimnospermas, y la clasificación propuesta por Mickel y Smith (2004) para pteridofitas y grupos afines. Los autores de las especies se abreviaron de acuerdo con Brummitt y Powell (1992).

Los especímenes se cotejaron en las colecciones del Herbario de la Facultad de Ciencias (FCME) y el Herbario Nacional del Instituto de Biología (MEXU), ambos de la UNAM. El material resultante de este trabajo está en proceso de ser depositado en el herbario FCME.

\section{Resultados}

Caracterización del bosque. El bosque de Lolotla se presenta en un piso altitudinal que va desde los 1050 a los $1580 \mathrm{~m}$. Presenta un relieve accidentado, con pendientes muy inclinadas y cañadas que albergan y protegen la vegetación de la insolación y de los fuertes vientos.

Los árboles del estrato arbóreo alto (dosel arbóreo) son pocos, pero algunos son corpulentos y con troncos que tienen diámetros que alcanzan los 65 y $70 \mathrm{~cm}$. Los individuos en este estrato llegan a medir más de $20 \mathrm{~m}$ de altura (algunos hasta $30 \mathrm{~m}$ ), como Dalbergia palo-escrito, Quercus germana, Quercus sartorii y Trophis mexicana). Otras especies presentes en este estrato son Clethra mexicana, Liquidambar macrophylla, Ostrya virginiana, Pinus greggii, P. pseudostrobus y Quercus eugeniifolia.

En el estrato arbóreo medio (10 a 20 m), los individuos en general presentan troncos con diámetros que alcanzan 30 y $45 \mathrm{~cm}$ de diámetro. Las especies más importantes son: Befaria laevis, Beilschmiedia mexicana, Carpinus caroliniana, Carya ovata var. mexicana, C. palmeri, Eugenia xalapensis, Inga huastecana, Ocotea helicterifolia, Prunus brachybotria, Quercus affinis, Q. castanea, Turpinia occidentalis y Vaccinium leucanthum.

El estrato arbóreo bajo, de menos de $10 \mathrm{~m}$ de alto, es muy diverso; los troncos de los árboles son muy delgados, alcanzando diámetros que van desde 3 hasta 12 $\mathrm{cm}$. Las especies más sobresalientes son: Cinnamomum effusum, Cornus disciflora, C. excelsa, Cyathea godmanii, 
Gaultheria acuminata, Ilex tolucana, Lonchocarpus caudatus, Lyonia squamulosa, Oreopanax xalapensis, Persea liebmannii, Prunus serotina ssp. capuli, Rapanea myricoides, Rhamnus longistyla, Trichilia havanensis, Viburnum ciliatum y Zanthoxylum xicense, entre otros.

El estrato arbustivo también está bien representado y está compuesto por especies como Baccharis trinervis, Bocconia frutescens, Cestrum fasciculatum, Eupatorium spp., Hoffmannia spp., Miconia spp., Palicourea padifolia, Piper auritum, Randia aculeata y Solanum spp. En sitios abiertos es frecuente la presencia de Cnidoscolus multilobus, que se ve favorecida por el disturbio.

Las plantas herbáceas terrestres que crecen en este bosque también son abundantes y algunas de ellas están favorecidas por la perturbación y la alta humedad ambiental. Entre ellas se encuentran representantes de varias familias, como Asplenium spp., Begonia spp., Coccocypselum cordifolium, Elaphoglosum spp., Equisetum myriochaetum, Lasiacis spp., Lobelia spp., Moussonia deppeana, Oxalis spp., Polypodium rosei, Selaginella spp., Tibouchina spp. y Pteridium sp. En los lugares más húmedos son frecuentes las orquídeas como Calanthe calanthoides, Cyclopogon luteo-albus, Dichaea glauca, Govenia spp. y Habenaria spp. En los lugares más perturbados son frecuentes las ciperáceas, comelináceas, gramíneas y asclepiadáceas, entre otras.

Entre las enredaderas y trepadoras, tanto herbáceas como leñosas, mejor representadas están: Blepharodon mucronatum, Canavalia villosa, Celastus pringlei, Clematis acapulcensis, Clitoria mexicana, Dioscorea ulinei, Ipomoea spp., Passiflora spp., Phaseolus spp., Smilax spp. y Vitis popenoei.

Las plantas epífitas crecen formando grupos sobre los troncos y las ramas de los árboles, en especial los compuestos por especies de bromelias, cactáceas, helechos, piperáceas y de orquídeas, como Aporocactus flagelliformis, Encyclia candollei, Epidendrum longipetalum, Isochilus aff. unilateralis, Peperomia spp., Phlebodium spp., Pleopeltis polylepis var. interjecta, Polypodium spp., Rhynchostele rossii, Stanhopea tigrina, Tillandsia spp. y Vittaria graminifolia.
Las parásitas son escasas, encontrándose generalmente en los lugares más perturbados; entre ellas están Cuscuta tinctoria, Phoradendrum falcatum y Struthanthus deppeanus.

En los márgenes de los principales ríos de la zona encontramos elementos de vegetación riparia, entre los cuales Platanus mexicana es la especie más conspicua.

En el área de estudio pueden diferenciarse principalmente dos vertientes, una norte y otra sur. Las laderas del bosque con exposición norte se caracterizan por ser las más húmedas, y en los sitios más conservados prosperanClethramexicana,Ingahuastecana,Liquidambar macrophylla, Quercus affinis, Q. germana, Q. sartorii, Ostrya virginiana y Turpinia occidentalis. En las zonas perturbadas de esta vertiente y más expuestas a la luz solar son frecuentes los individuos de Gaultheria acuminata, Pinus pseudostrobus y Vaccinium leucanthum. En la vertiente sur son frecuentes Befaria laevis, Beilschmedia mexicana, Clethra mexicana, Eugenia xalapensis, Inga huastecana, Quercus affinis, Q. castanea, Q. eugeniifolia, Q. germana, $Q$. sartorii y Vaccinium leucanthum.

Composición florística. Se ha conjuntado una lista florística de las plantas vasculares del área, compuesta por 103 familias, 260 géneros, 359 especies y 11 taxa subespecíficos (Apéndice 1). El grupo de plantas vasculares más numeroso es el de las angiospermas $(90.6 \%$ de las especies), seguido de las pteridofitas y grupos afines (8.9\%); las gimnospermas están representadas por pocas especies $(1.1 \%)$. Las dicotiledóneas son las mejor representadas $(71.8 \%$ de las especies) a nivel de familia, género, especie y taxón subespecífico (Cuadro 1).

Las familias más diversas en cuanto a número de géneros se muestran en la figura 2; resaltan las compuestas, leguminosas y orquídeas. Las familias más diversas en cuanto a número de especies son las compuestas, orquídeas, leguminosas, solanáceas, rubiáceas y polipodiáceas (Fig. 3).

Respecto al número de especies por forma de vida, las hierbas son las mejor representadas con 147 especies (40.9\%), seguidas por los árboles con 78 (21.7\%), arbustos

Cuadro 1. Riqueza de plantas vasculares.

\begin{tabular}{lllll}
\hline Grupo & Número de familias & Número de géneros & Número de especies & Número de subespecies \\
\hline Pteridophyta y afines & $11(10.7 \%)$ & $20(7.6 \%)$ & $32(8.9 \%)$ & $1(9 \%)$ \\
Gimnospermae & $2(1.9 \%)$ & $2(0.7 \%)$ & $4(1.1 \%)$ & 0 \\
Dicotyledonae & $79(76.7 \%)$ & $191(73.0 \%)$ & $258(71.8 \%)$ & $9(81 \%)$ \\
Monocotyledonae & $11(10.7 \%)$ & $47(18.0 \%)$ & $65(18.1 \%)$ & $1(9 \%)$ \\
Total & $103(100 \%)$ & $260(100 \%)$ & $359(100 \%)$ & $11(100 \%)$ \\
\hline
\end{tabular}




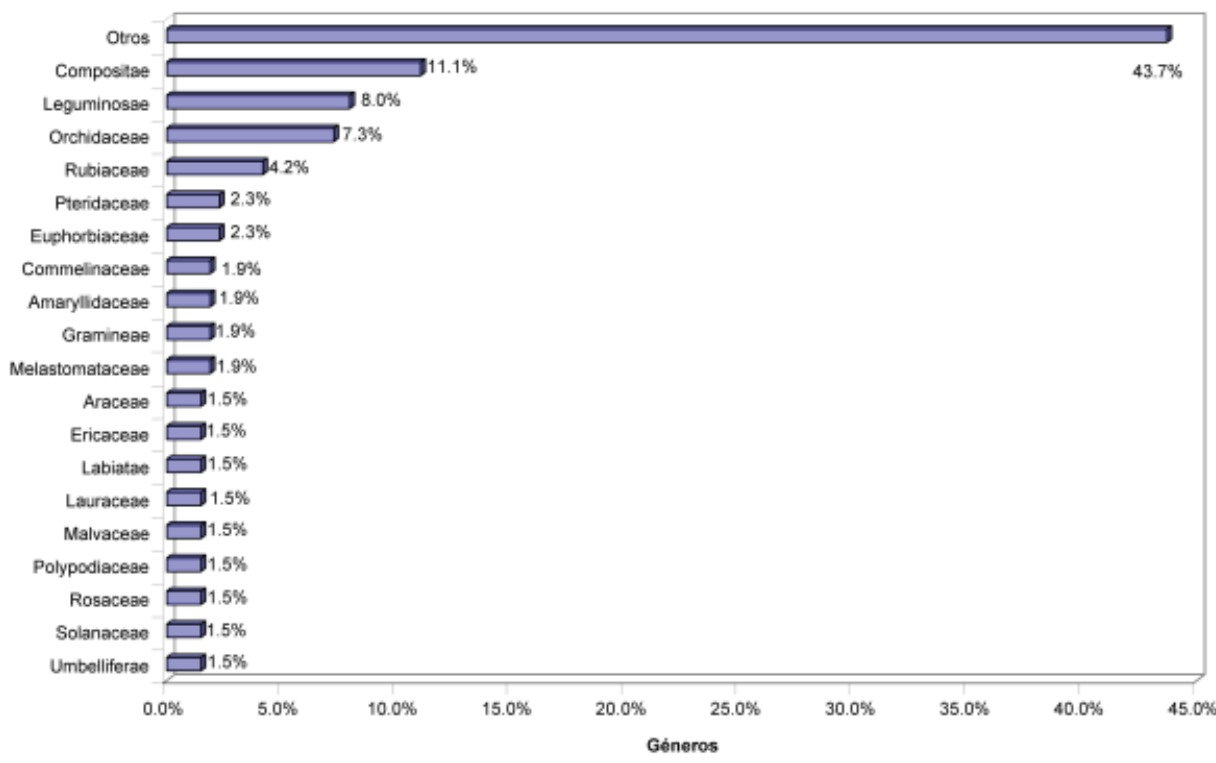

Figura 2. Familias más diversas en cuanto a número de géneros.

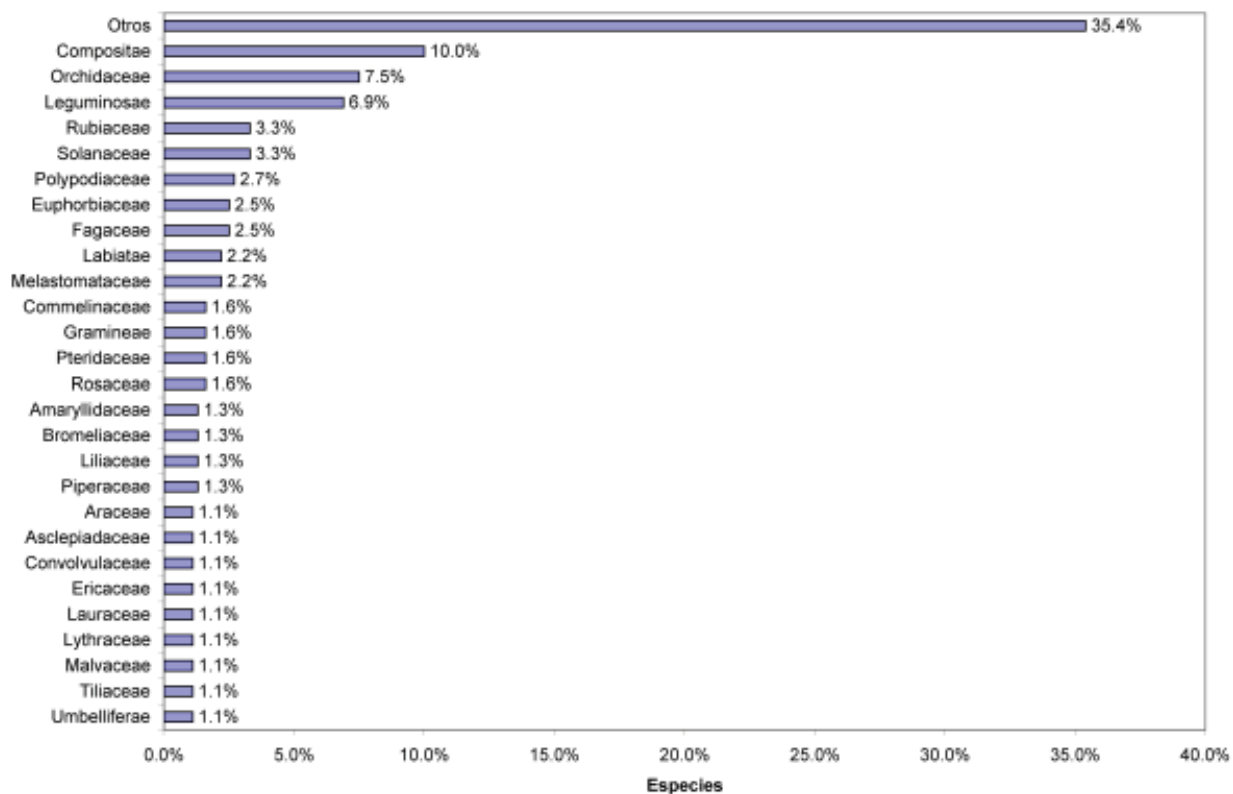

Figura 3. Familias más diversas en cuanto a número de especies.

con $62(17.2 \%)$, bejucos con 35 (9.7\%), epífitas con 34 (9.4\%) y parásitas con 3 (0.8\%) (Fig. 4).

\section{Discusión y conclusiones}

El conocimiento florístico del bosque del municipio de
Lolotla era inexistente, por lo que este trabajo representa un primer esfuerzo para obtener una lista florística del área.

El bosque del municipio de Lolotla ha sido severamente modificado por sus habitantes, siendo la ganadería, agricultura de temporal y extracción de leña las actividades que más lo han afectado, lo que se ve 


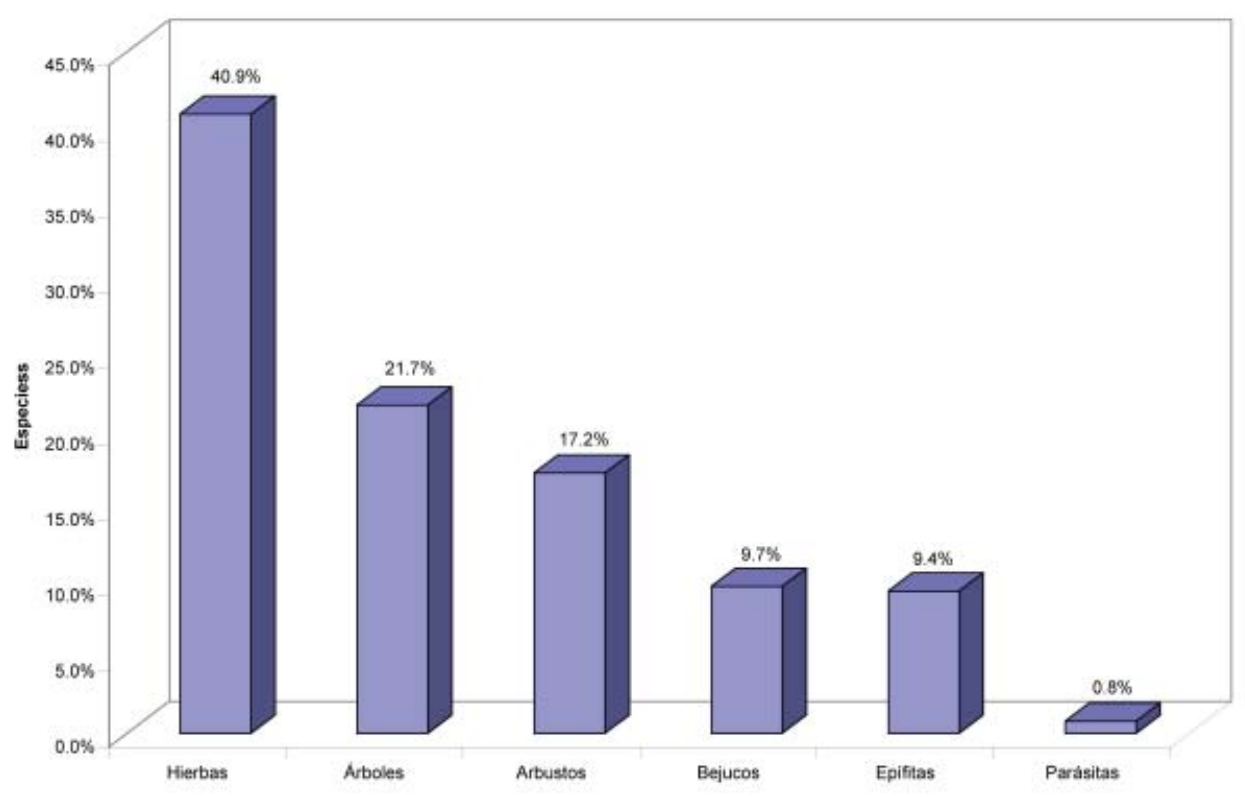

Figura 4. Número de especies por forma de vida. En la categoría de bejucos se incluyen enredaderas y trepadoras; en la categoría de hierbas se excluye a las epífitas.

reflejado en la escasez de individuos arbóreos altos con diámetros grandes. Los estudios de vegetación y estructura poblacional de Escutia (2004) sugieren que este bosque está en proceso de regeneración; no obstante, se considera que el bosque mesófilo de montaña de Monte Grande aún posee una flora rica y diversa constituida por 359 especies de plantas vasculares. Este bosque está asentado en sitios de topografía abrupta y pendientes fuertes, y pertenece a la Región Terrestre Prioritaria 102, llamada Bosques mesófilos de la Sierra Madre Oriental (Conabio, 2000). Dado al manejo que ha tenido el bosque, faltan algunas familias representativas del bosque mesófilo prístino y maduro, como son Magnoliaceae, Podocarpaceae, Styracaceae y Symplocaceae, entre otras.

De las familias que Rzedowski (1996) considera que prosperan preferentemente en el bosque mesófilo de montaña mexicano, en Monte Grande se presentan las siguientes: Aquifoliaceae, Begoniaceae, Clethraceae, Cornaceae, Cyatheaceae, Gesneriaceae, Lauraceae, Myrsinaceae, Orchidaceae, Piperaceae, Selaginellaceae, Staphyleaceae y Theaceae, esto es, el 39\%. Las familias Hamamelidaceae y Nyssaceae, con representantes exclusivos o casi exclusivos en este tipo de vegetación, también están representadas en la zona de estudio.

Algunos géneros de árboles cuantitativamente importantes en los bosques mesófilos de montaña (Rzedowski, 1996) y presentes en Monte Grande son: Alnus, Carpinus, Carya, Clethra, Cleyera, Cornus, Dalbergia, Dendropanax, Liquidambar, Nyssa, Oreopanax, Persea,
Prunus, Quercus y Ternstroemia, mismos que poseen especies características de este tipo de vegetación. Otros géneros de plantas vasculares representadas en Monte Grande que poseen especies características de este tipo de vegetación son: Anthurium, Begonia, Chamaedorea, Elaphoglossum, Encyclia, Epidendrum, Hoffmannia, Miconia, Peperomia, Pilea, Polypodium, Rondeletia, Saurauia, Selaginella y Tillandsia.

A continuación se anotan las categorías de riesgo de la Norma Oficial Mexicana NOM-059-ECOL-2001 (SEMARNAT, 2002) y las especies que están dentro de ellas : a) en peligro de extinción (especies cuyas áreas de distribución o tamaño de sus poblaciones en el territorio nacional han disminuido drásticamente poniendo en riesgo su viabilidad biológica en todo su hábitat natural): Laelia anceps; b) amenazadas (especies o poblaciones de éstas que podrían llegar a encontrarse en peligro de desaparecer a corto o mediano plazo, si siguen operando los factores que inciden negativamente en su viabilidad): Carpinus caroliniana, Ceratozamia mexicana, Disocactus phyllanthoides, Mormodes maculata spp. unicolor Rynchostele rossii y Stanhopea tigrina; c) sometidas a protección especial (especies o poblaciones que podrían llegar a encontrarse amenazadas por factores que inciden negativamente en su viabilidad, por lo que se determina la necesidad de propiciar su recuperación y conservación o la recuperación y conservación de poblaciones de especies asociadas): Aporocactus flagelliformis, Cyathea fulva, Ostrya virginiana y Prosthechea vitellina. 
Otras especies con distribución restringida en los bosques mesófilos de montaña mexicanos y que están representadas en Monte Grande son Carya palmeri var. mexicana y Dalbergia palo-escrito, mismas que sugerimos sean evaluadas para su posible inclusión en la Norma Oficial.

Los bosques de la Huasteca hidalguense conforman fracciones de un manchón más extenso que se han venido fragmentando con el transcurso del tiempo por diferentes causas (Alcántara y Luna, 2001). En particular, el bosque de Monte Grande ha sido manejado intensivamente, sobre todo en los últimos años, por lo que actualmente está constituido por un mosaico complejo de diferentes estados de sucesión de bosque. Los principales usos que se le han dado al bosque de la zona han sido los cultivos de cítricos, frijol y maíz, ganadería (con la subsecuente formación de potreros) y extracción de madera. La ausencia en el bosque de árboles altos con diámetros grandes es evidente, lo que da como consecuencia que especies pertenecientes a familias propias de lugares perturbados sean numerosas: Leguminosae, Solanaceae y Compositae, entre otras. Hay especies que pueden considerarse como parte del bosque alterado, como Cnidoscolus multilobus, Commelina diffusa, Crataegus mexicana, Homolepis glutinosa, Phytolacca icosandra, Pteridium sp. y Rubus eriocarpus, entre otras, y algunas que se benefician por el aclareamiento del bosque, pero que pueden ser parte del bosque conservado: Alnus jorullensis, Cornus disciflora, Saurauia scabrida y Smilax spp.

\section{Agradecimientos}

Raúl Contreras y Jaime Jiménez hicieron sugerencias para mejorar el manuscrito. Agradecemos a las siguientes personas que nos ayudaron en el trabajo de campo: Raúl Contreras Medina, Mauricio Mora Jarvio, Maribel Paniagua, Gimena Pérez Ortega y Alberto González Zamora. Recibimos ayuda en la determinación de material de Susana Valencia Ávalos (Fagaceae), Martha Martínez Gordillo (Euphorbiaceae), Ramiro Cruz Durán (Leguminosae), Gerardo Salazar Chávez (Orchidaceae), Frank Almeida (Melastomataceae), Adolfo Espejo y Ana Rosa López Ferrari (Bromeliaceae), Oswaldo Tellez (Dioscoreaceae), Beatriz González Hidalgo (compuestas) y José Luis Villaseñor (compuestas y familias varias). Este trabajo fue financiado por el fondo sectorial de CONACYT, proyecto SEMARNAT-2004-C01-311.

\section{Literatura citada}

Alcántara, O. e I. Luna. 1997. Florística y análisis biogeográfico del bosque mesófilo de montaña de Tenango de Doria, Hidalgo, México. Anales del Instituto de Biología de la Universidad Nacional Autónoma de México, Serie Botánica 68: 57-106.

Alcántara, O. e I. Luna. 2001. Análisis florístico de dos áreas con bosque mesófilo de montaña en el estado de Hidalgo, México: Eloxochitlán y Tlahuelompa. Acta Botanica Mexicana 54: 51-87.

Brummitt, R. K. y C. E Powell (eds.). 1992. Authors of plant names. Royal Botanic Gardens, Kew. 732 p.

CETENAL. 1976. Fotografías aéreas. ZIIA Escala 1:50,000. Febrero 1976.

Challenger, A. 1998. Utilización y conservación de los ecosistemas terrestres de México. Pasado, presente y futuro. Comisión Nacional para el Conocimiento y Uso de la Biodiversidad; Instituto de Biología, UNAM, y Agrupación Sierra Madre, México, D.F. 847 p.

Conabio-Estadigrafía. 1997. Mapas de climas. F047. Escala 1:1 000 000. Comisión Nacional para el Conocimiento y Uso se la Biodiversidad, México.

Conabio. 2000. Regiones terrestres prioritarias. Escala 1 000 000. Comisión Nacional para el Conocimiento y Uso de la Biodiversidad, México.

Engler, A. y L. Diels. 1936. Syllabus der Pflanzenfamilien, $11^{\mathrm{a}}$. edición, Berlin.

Escutia, J. A. 2004. Análisis estructural del bosque mesófilo de montaña de Monte Grande Lolotla, Hidalgo, México. Tesis Facultad de Ciencias, Universidad Nacional Autónoma de México. México, D.F. 98 p.

García, E. 2004. Modificaciones al sistema de clasificación climática de Köppen, $5^{\text {a }}$ edición, Serie Libros Núm. 6. Instituto de Geografía, Universidad Nacional Autónoma de México, México, D.F. 90 p.

INEGI (Instituto Nacional de Estadística, Geografía e Informática). 1989. Carta topográfica Molango 1: 50000 F14D51. México.

INEGI (Instituto Nacional de Estadística, Geografía e Informática). 1992. Síntesis geográfica del estado de Hidalgo. México. 134 p.

INEGI (Instituto Nacional de Estadística, Geografía e Informática). 1995. Anuario estadístico del estado de Hidalgo. Secretaría de Gobernación, México, D.F.

INEGI (Instituto Nacional de Estadística, Geografía e Informática). 1995. Fotografías aéreas F-1411. Escala 1:75,000. Diciembre 1995.

INEGI (Instituto Nacional de Estadística, Geografía e Informática). 1999. Carta edafológica. Escala 1:250,000. Pachuca F1411, 2a impresión. México, D.F.

Lot, A. y F. Chiang. 1986. Manual de Herbario. Consejo Nacional de la Flora de México, México, D.F. 142 p.

Luna, I., S. Ocegueda y O. Alcántara. 1994. Florística y notas biogeográficas del bosque mesófilo de montaña 
del municipio de Tlanchinol, Hidalgo, México. Anales del Instituto de Biología. Universidad Nacional Autónoma de México, Serie Botánica 65: 31- 62.

Luna, I. y O. Alcántara. 2004. Florística del bosque mesófilo de montaña de Hidalgo. In Biodiversidad de la Sierra Madre Oriental, I. Luna, J. J. Morrone y D. Espinosa (eds.). Conabio-UNAM, México, D.F., pp. 169-192.

Mayorga, R., I. Luna y O. Alcántara. 1998. Florística del bosque mesófilo de montaña de Molocotlán, MolangoXochicoatlán, Hidalgo, México. Boletín de la Sociedad Botánica de México 63: 101-119.

Meave, J., M. A. Soto, L.M. Calvo, H. Paz y S. Valencia. 1992. Análisis sinecológico del bosque mesófilo de montaña de Omiltemi, Guerrero. Boletín de la Sociedad Botánica de México 52: 31-77.

Mickel, J. T. y A. R. Smith. 2004. The pteridophytes of Mexico. Memoires of the New York Botanical Garden 88: $1-1054$.

Miranda, F. y A. J. Sharp. 1950. Characteristics of the vegetation in certain temperate regions of eastern Mexico. Ecology 31: 313-333.

Ortega, F. y G. Castillo. 1996. El bosque mesófilo de montaña y su importancia forestal. Ciencias 43: 32-39.

Paray, L. 1946. Exploraciones botánicas en el norte del estado de Puebla. Boletín de la Sociedad Botánica de México 4: 10-12.

Paray, L. 1949. Exploraciones botánicas en el estado de
Hidalgo. Boletín de la Sociedad Botánica de México 8: $1-7.0$

Puig, H. 1976. Végétation de la Huasteca, Mexique. Mission Archéologique et Ethnologique Française au Mexique. México, D.F. 531 p.

Rzedowski, J. 1978. Vegetación de México. Limusa. México. $432 \mathrm{p}$.

Rzedowski, J. 1996. Análisis preliminar de la flora vascular de los bosques mesófilos de montaña de México. Acta Botanica Mexicana 35: 25-44.

SEMARNAT (Secretaría del Medio Ambiente y Recursos Naturales). 2002. Norma Oficial Mexicana NOM-059ECOL-2001, protección ambiental-especies nativas de México y de flora y fauna silvestres- categorías de riesgo y especificaciones para su inclusión, exclusión o cambio- lista de especies en riesgo. Diario Oficial de la Federación, México, 6 de marzo, pp. 1-80.

SCT (Secretaría de Comunicaciones y Transportes). 1987. Mapa de carreteras del estado de Hidalgo. Escala 1:400,000.

SP (Secretaría de la Presidencia). 1970. Carta de climas. Pachuca 14Q-(IV). México D.F.

SPP (Secretaría de Programación y Presupuesto). 1983a. Carta geológica. Escala 1:250,000 Pachuca F1411. México, D.F.

SPP (Secretaría de Programación y Presupuesto). 1983b. Carta hidrológica de aguas superficiales. Escala 1:250,000 Pachuca F1411. México, D.F.

Apéndice 1. Lista florística del bosque mesófilo de montaña de "Monte Grande," Lolotla, Hidalgo, México.

$\mathrm{Ab}=$ árbol bajo, $\mathrm{Am}$ = árbol mediano, $\mathrm{Aa}=$ árbol alto, $\mathrm{Ar}=$ arbusto, $\mathrm{H}=$ hierba terrestre, $\mathrm{E}=$ epífita, $\mathrm{B}=$ enredaderas y trepadoras, $\mathrm{P}=$ parásita.

\section{PTERIDOPHYTA Y GRUPOS AFINES}

\section{ASPLENIACEAE}

H. Asplenium auriculatum Sw.

H. A. blepharophorum Bertol.

\section{BLECHNACEAE}

H. Blechnum schiedeanum (Schltdl. ex Presl) Hieron.

H. Woodwardia martinezii Maxon ex Weath.

\section{CYATHEACEAE}

Ab. Cyathea fulva (M. Martens et Galeotti) Fée

Ab. C. godmanii (Hook.) Domin

Ab. C. schiedeana (C. Presl) Domin

\section{DENNSTAEDTIACEAE}

H. Pteridium sp.

\section{DRYOPTERIDACEAE}

H. Elaphoglossum sartorii (Liebm.) Mickel

H. E. vestitum (Schltldl. et Cham.) Schott ex T. Moore

EQUISETACEAE

H. Equisetum myriochaetum Schltdl. et Cham.

\section{OPHIOGLOSSACEAE}

H. Botrychium virginianum (L.) Sw.

\section{POLYPODIACEAE}

E. Campyloneurum angustifolium (Sw.) Fée

E. Phlebodium areolatum (Humb. et Bonpl. ex Willd.) J. Sm.

E. Ph. pseudoaureum (Cav.) Lellinger

E. Pleopeltis polylepis (Roemer ex Kunze) T. Moore var. interjecta (Weath.) E.A. Hooper 
E. Polypodium cryptocarpon Fée

E. P. longepinnulatum E.Fourn.

E. P. plebeium Schltdl. et Cham.

E. P. polypodioides (L.) Watt

E. P. rhodopleuron Kunze

H. $P$. rosei Maxon

\section{PTERIDACEAE}

H. Adiantopsis radiata (L.) Fée

H. Adiantum andicola Liebm.

H. Llavea cordifolia Lag.

H. Pityrogramma ebenea (L.) Proctor

H. Pteris orizabae M.Martens et Galeottii

E. Vittaria graminifolia Kaulf.

\section{SCHIZAEACEAE}

H. Anemia phyllitidis (L.) Sw.

\section{SELAGINELLACEAE}

H. Selaginella harrisii Underw. et Hieron.

H. S. sertata Spring

H. S. wrightii Hieron.

\section{GYMNOSPERMAE}

\section{CYCADACEAE}

Ar. Ceratozamia mexicana Brongn.

\section{PINACEAE}

Aa. Pinus greggii Engelm. ex Parl. Am. P. oocarpa Schiede ex Schltdl. Aa. P. pseudostrobus Lindl.

\section{ANGIOSPERMAE}

\section{MONOCOTYLEDONAE}

\section{AMARYLLIDACEAE}

B. Bomarea acutifolia (Link et Otto) Herb.

H. Furcraea sp.

H. Hypoxis decumbens L.

H. Manfreda brachystachys (Cav.) Rose

$\mathrm{H}$. Zephyranthes lindleyana Herb.

\section{ARACEAE}

E. Monstera deliciosa Liebm.

E. Syngonium sp.

H. Anthurium sp.

H. Arisaema sp.

\section{BROMELIACEAE}

H. Pitcairnia ringens Klotzsch

E. Tillandsia bartramii Elliott

E. T. deppeana Steud.

E. T. gymnobotrya Baker

E. T. viridiflora (Beer) Baker

\section{COMMELINACEAE}

H. Aneilema sp.

H. Commelina diffusa Burm.f.

H. Gibasis pellucida (M.Martens et Galeotti) Hunt

H. Tinantia erecta (Jacq.) Schltdl.

H. Tripogandra serrulata (Vahl) Handlos

H. T. amplexicaulis (Klotzsch ex C.B. Clarke) Woodson

\section{CYPERACEAE}

H. Cyperus odoratus L.

H. Rhynchospora aristata Baeckeler

H. $R$. radicans (Schltdl. et Cham.) H. Pfeiff.

\section{DIOSCOREACEAE}

B. Dioscorea ulinei Greenm. ex R. Knuth

\section{GRAMINEAE}

H. Bouteloua sp.

H. Homolepis glutinosa (Sw.) Zuloaga et Soderstrom

H. Lasiacis divaricata (L.) Hitchc.

H. L. procerrima (Hack.) Hitchc.

H. Muhlenbergia sp.

H. Paspalum notatum Flüggé

\section{IRIDACEAE}

H. Orthrosanthus exsertus (R.C.Foster) Ravenna

H. Tigridia pavonia (L.f.) DC.

\section{LILIACEAE}

H. Echeandia mexicana Cruden

B. Smilax aristolochiifolia Mill.

B. S. domingensis Willd.

B. S. mollis Humb. et Bonpl. ex Willd.

B. S. tomentosa Kunth

\section{ORCHIDACEAE}

E. Anathallis platystylis (Schltr.) Pridgeon et M.W. Chase

H. Bletia gracilis Lodd.

H. B. neglecta Sosa

H. Calanthe calanthoides (A. Rich. et Galeotti) Hamer et Garay

H. Cyclopogon aff. luteo-albus (A.Rich. et Galeotti) Schltr.

H. C. luteo-albus (A. Rich. et Galeotti) Schltr.

H. Dichaea glauca (Sw.) Lindl.

E. Encyclia candollei (Lindl.) Schltr. 
E. E. aff. candollei (Lindl.) Schltr.

E. Epidendrum longipetalum A. Rich. et Galeotti

E. Euchile mariae (Ames) Withner

H. Govenia aff. mutica Rchb.f.

H. G. mutica Rchb.f.

H. G. praecox Salazar et E.W. Greenw.

H. Habenaria floribunda Lindl.

H. H. jaliscana S. Watson

E. Isochilus aff. unilateralis Rob.

E. Laelia anceps Lindl.

H. Malaxis brachyrrhynchos (Rchb.f.) Ames

H. M. excavata (Lindl.) O. Kuntze

E. Mormodes maculata Hook. f. var. unicolor L.O.

Williams

E. Prosthechea ochracea (Lindl.) W.E. Higgins

E. P. vitellina (Lindl.) W.E. Higgins

E. Rhynchostele aff. rossii (Lindl.) Soto Arenas et Salazar

E. $R$. rossii (Lindl.) Soto Arenas et Salazar

E. Stanhopea tigrina Bateman et Lindl.

E. Stelis ornata (Rchb.f.) Pridgeon et M.W. Chase

PALMAE

H. Chamaedorea tepejilote Liebm.

\section{DICOTYLEDONAE}

\section{ACANTHACEAE}

H. Aphelandra speciosa Brandegee

H. Ruellia harveyana Stapf

\section{AMARANTHACEAE}

H. Amaranthus hybridus L.

H. Iresine diffusa Humb. et Bonpl. ex Willd

\section{ANACARDIACEAE}

B. Rhus radicans $\mathrm{L}$.

\section{APOCYNACEAE}

B. Mandevilla subsagittata (Ruiz et Pav.) Woodson

\section{AQUIFOLIACEAE}

Am. Ilex discolor Hemsl.

Ab. I. tolucana Hemsl.

\section{ARALIACEAE}

Am. Dendropanax arboreus (L.) Decne. et Planch. Ab. Oreopanax xalapensis (Kunth) Decne. et Planch.

\section{ASCLEPIADACEAE}

H. Asclepias curassavica L.

H. A. ovata M. Martens et Galeotti
B. Blepharodon mucronatum (Schltdl.) Decne.

B. Oxypetalum cordifolium (Vent.) Schltr.

\section{BEGONIACEAE}

H. Begonia incarnata Link et Otto

H. B. manicata Brongn.

H. B. multistaminea Burt-Utley

BERBERIDACEAE

Ab. Berberis lanceolata Benth.

\section{BETULACEAE}

Ab. Alnus jorullensis Kunth ssp. lutea Furlow

Am. Carpinus caroliniana Walter

Aa. Ostrya virginiana (Mill.) K.Koch

BIGNONIACEAE

B. Pithecoctenium crucigerum (L.) A. Gentry

BORAGINACEAE

Ar. Cordia spinescens L.

CACTACEAE

E. Aporocactus flagelliformis (L.) Lem.

E. Disocactus phyllanthoides (DC.) Barthlott

\section{CAMPANULACEAE}

H. Diastatea micrantha (Kunth) McVaugh

H. Lobelia laxiflora Kunth

H. L. schmitzii E. Wimm.

CAPPARIDACEAE

H. Gynadropsis speciosa DC.

\section{CAPRIFOLIACEAE}

Ab. Sambucus nigra var. canadensis (L.) B.L. Turner Ab. Viburnum ciliatum Greenm.

\section{CARYOPHYLLACEAE}

H. Stellaria cuspidata Willd.

CELASTRACEAE

B. Celastrus pringlei Rose

Ab. Perrottetia ovata Hemsl.

\section{CLETHRACEAE}

Ab. Clethra macrophylla M. Martens et Galeotti

Aa. C. mexicana A. DC.

Ab. C. pringlei S.Watson

COMPOSITAE

H. Ageratum corymbosum Zuccagni 
Ar. Alloispermum integrifolium (DC.) H. Rob.

H. Aster potosinus Gray

H. A. subulatus Michx.

Ar. Archibacharis hirtella (DC.) Heering

Ar. A. schiedeana (Benth.) J.D. Jacks.

Ar. Baccharis trinervis Pers.

H. Bidens bigetovii Gray

H. B. odorata Cav.

Ar. Calea ternifolia Kunth var. ternifolia

Ar. Coreopsis petrophiloides B.L. Rob. et Greenm.

H. Dahlia coccinea Cav.

H. D. sorensenii H.V.Hansen et J.P. Hjerting

H. Elephantopus mollis Kunth

Ar. Eupatorium leucocephalum Benth.

Ar. E. ligustrinum DC.

Ar. E. platyphyllum B.L. Rob.

H. Gnaphalium ehrenbergianum Sch. Bip

H. Jaegeria hirta (Lag.) Less.

Ar. Kyrsteniopsis sp.

H. Lactuca sp.

H. Leiboldia serrata Gleason

Ar. Montanoa sp.

H. Packera sanguisorbae (DC.) C. Jeffrey

Ar. Perymenium ovalifolium (A. Gray) B.L. Turner

Ar. Roldana aschenborniana (Schauer) H.Rob. et Brettell

H. Senecio salignus DC.

Ar. Sinclairia deppeana Rydb.

H. Stevia jorullensis Kunth

H. S. ovata Willd.

H. Telanthophora grandifolia (Less.) H. Rob. et Bretell var. grandifolia

Ar. Verbesina hypomalaca B.L. Rob. et Greenm.

Ar. V. virgata Cav.

Ar. Vernonia deppeana Less.

Ar. Vernonanthura patens (Kunth) H. Rob.

Ar. Viguiera sp.

\section{CONVOLVULACEAE}

P. Cuscuta tinctoria Mart. ex Engelm.

B. Ipomoea funis Schltdl. et Cham.

B. I. purpurea (L.) Roth

B. I. phillomega (Vell.) House

\section{CORNACEAE}

Ab. Cornus disciflora Moc. et Sessé ex DC.

Ab. C. excelsa Kunth

CRASSULACEAE

H. Echeveria aff. guatemalensis Rose

CUCURBITACEAE

B. Melothria pendula L.
DILLENIACEAE

Ab. Saurauia scabrida Hemsl.

ELAEOCARPACEAE

Ab. Sloanea sp.

\section{ERICACEAE}

Am. Befaria laevis Benth.

Ab. Gaultheria acuminata Schltdl. et Cham.

Ab. Lyonia squamulosa M. Martens et Galeotti

Am. Vaccinium leucanthum Schltdl.

\section{EUPHORBIACEAE}

Ar. Acalypha sp.

Ar. Cnidoscolus multilobus (Pax) I.M. Johnst.

Ab. Croton draco Schltdl.

H. Euphorbia dentata Michx.

H. E. graminea Jacq.

Ar.E . hyssopifolia L.

H. E. orizabae Boiss.

Ab.Gymnanthes sp.

H. Phyllanthus sp.

FAGACEAE

Am.Quercus affinis Scheidw.

Ab. Q. aff. laurina Kunth

Am.Q. castanea Née

Ab. Q. deserticola Trel.

Aa. Q. aff. eugeniifolia Liebm.

Aa. Q. eugeniifolia Liebm.

Aa. Q. germana Schltdl.

Am.Q. polymorpha Schltdl. et Cham.

Aa. Q. sartorii Liebm.

FLACOURTIACEAE

Ar. Xylosma flexuosum (Kunth) Hemsl.

GERANIACEAE

H. Geranium seemannii Peyr.

\section{GESNERIACEAE}

H. Achimenes grandiflora DC.

H. Moussonia deppeana (Schltdl. et Cham.) Hanst.

GUTTIFERAE

H. Ascyrum hypericoides L.

HAMAMELIDACEAE

Aa. Liquidambar macrophylla Oerst.

JUGLANDACEAE 
Am. Carya ovata (Mill.) K.Koch var. mexicana (Engelm.) W.E. Manning

Am. C. palmeri W.E. Manning

\section{LABIATAE}

H. Hyptis capitata Jacq.

H. H. mutabilis Briq.

H. Ocimum selloi Benth.

H. Salvia heliantemifolia Benth.

H. S. involucrata Cav.

H. S. membranacea Benth.

H. S. mexicana L. var. mexicana

H. Stachys boraginoides Cham. et Schltdl.

\section{LAURACEAE}

Am. Beilschmiedia mexicana (Mez) Kosterm.

Ab. Cinnamomum effusum (Meissn.) Kosterm.

Am. Ocotea helicterifolia (Meissn.) Hemsl.

Ab. Persea liebmannii Mez

\section{LEGUMINOSAE}

Ar. Amicia zygomeris DC.

B. Canavalia villosa Benth.

B. Centrosema sp.

H. Chamaecrista chamaecristoides (Coll.) Greene var. chamaecristoides

H. C. glandulosa (L.) Greene

B. Clitoria mexicana Link

H. Crotalaria acapulcensis Hook. et Arn.

H. C. rotundifolia (Walter) Walter ex J.F. Gmel.

Ab. Dalbergia glomerata Hemsl.

Aa. D. palo-escrito Rzed. et Guridi-Gómez

H. Desmodium caripense (Kunth) G. Don

H. D. plicatum Schltdl. et Cham.

Ab. Erythrina americana Mill.

Am. Inga huastecana M. Sousa

Ab. Leucaena collinsii Britton et Rose

Ab. Lonchocarpus caudatus Pittier

Ab. Lysiloma microphylla Benth.

Ar. Mimosa albida Humb. et Bonpl. ex Willd.

B. Phaseolus coccineus L.

B. P. vulgaris L.

Ab. Platymiscium lasiocarpum Sandwith

Ar. Senna pendula (Humb. et Bonpl. ex Willd.) H.S. Irwin et Barneby

Ar. S. septemtrionalis (Viv.) H.S. Irwin et Barneby

B. Vigna vexillata (L.) A. Rich.

Ab. Zapoteca portoricensis (Jacq.) H.M. Hern.

\section{LORANTHACEAE}

P. Phoradendron falcatum (Cham. et Schltdl.) Trel.

P. Struthanthus deppeanus (Schltdl. et Cham.) Blume

\section{LYTHRACEAE}

H. Cuphea angustifolia Jacq. ex Koehne

H. C. jorullensis Kunth

H. C. racemosa (L.f.) Spreng.

H. C. wrightii A. Gray

MALPIGHIACEAE

B. Heteropteris brachiata (L.) DC.

MALVACEAE

H. Anoda cristata (L.) Schltdl.

Ar. Malvaviscus arboreus Cav.

H. Pavonia schiedeana Steud.

H. Sida rhombifolia L.

\section{MELASTOMATACEAE}

H. Arthrostemma ciliatum Pav. ex D. Don

Ar. Conostegia xalapensis (Bonpl.) D. Don

Ar. Leandra cornoides (Schltdl. et Cham.) Cogn.

Ar. Miconia mexicana (Bonpl.) Naudin

Ar. M. moorei Wurdack

Ar. M. sylvatica (Schltdl.) Naudin

H. Tibouchina mexicana Cogn.

H. T. naudiniana Cogn.

MELIACEAE

Ab. Trichilia havanensis Jacq.

MENISPERMACEAE

B. Cissampelos pareira L.

MORACEAE

Aa. Trophis mexicana (Liebm.) Bureau

MYRICACEAE

Ar. Myrica cerifera L.

MYRSINACEAE

Ar. Ardisia sp.

Ab. Rapanea myricoides (Schltdl.) Lundell

MYRTACEAE

Ab. Eugenia oerstediana O. Berg

Am. E. xalapensis (Kunth) DC.

NYSSACEAE

Ab. Nyssa sylvatica Marshall

OLEACEAE

Ab. Forestiera reticulata Torr.

Ab. Osmanthus americanus (L.) Benth. ex Hook.f.

ONAGRACEAE 
H. Lopezia racemosa Cav. var. racemosa

H. Oenothera laciniata Hill

OXALIDACEAE

H. Oxalis dimidiata Donn. Sm.

H. O. rhombifolia Jacq.

PAPAVERACEAE

Ar. Bocconia frutescens L.

PASSIFLORACEAE

B. Passiflora capsularis L.

B. P. sicyoides Schltdl. et Cham.

B. P. subpeltata Ortega

\section{PHYTOLACCACEAE}

H. Phytolacca icosandra L.

\section{PIPERACEAE}

E. Peperomia collocata Trel.

E. P. quadrifolia (L.) Kunth

Ar. Pothomorphe umbellata (L.) Miq.

Ar. Piper auritum Kunth

Ar. P. fraguanum Trel.

PLANTAGINACEAE

H. Plantago australis Lam.

PLATANACEAE

Aa. Platanus mexicana Moric.

POLYGALACEAE

Ar. Monnina xalapensis Kunth

\section{POLYGONACEAE}

H. Polygonum acre Lam.

H. P. punctatum Elliot

H. Rumex obtusifolius L.

\section{RANUNCULACEAE}

B. Clematis acapulcensis Hook. et Arn.

H. Thalictrum pubigerum Benth.

\section{RHAMNACEAE}

Ab. Rhamnus longistyla C.B. Wolf

\section{ROSACEAE}

H. Acaena elongata L.

Ab. Crataegus mexicana Moc. et Sessé

Am. Prunus brachybotria Zucc.

Am. P. samydoides Schltdl.

Ab. P. serotina Ehrb. ssp. capuli (Cav.) McVaugh
Ar. Rubus eriocarpus Liebm.

RUBIACEAE

H. Borreria laevis Griseb.

H. Bouvardia ternifolia (Cav.) Schltdl.

H. Coccocypselum cordifolium Nees et Mart.

H. Crusea longiflora (Willd. ex Roem et Schult.) W.R. Anderson

Ar. Hoffmania aff. excelsa (Kunth) K. Schum.

Ar. H. orizabensis Standl.

H. Houstonia sp.

Ar. Palicourea padifolia (Willd. ex Roem. et Schult.) C.M.

Taylor et Lorence

Ar. Psychotria sp.

Ar. Randia aculeata L.

Ar. Rondeletia capitellata Hemsl.

H. Spermacoce tenuior L.

RUTACEAE

Ab. Zanthoxylum xicense Miranda

SAPINDACEAE

B. Serjania hispida Standl. et Steyerm.

\section{SAPOTACEAE}

Ab. Sideroxylon portoricense Urb. var. minutiflorum (Pittier) T.D. Penn.

\section{SCROPHULARIACEAE}

H. Lamourouxia multifida Kunth

B. Maurandya erubescens (D.Don) Gray

SIMAROUBACEAE

Ar. Picramnia xalapensis Planch.

SOLANACEAE

Ar. Brugmansia x candida Pers.

Ar. B. suaveolens (Humb. et Bonpl. ex Willd.) Bercht. et J. Presl

Ar. Cestrum faciculatum (Schltdl.) Miers

Ar. C. nocturnum L.

Ar. Cyphomandra betacea (Cav.) Sendtn.

Ab. Solanum aligerum Schltdl.

B. S. appendiculatum Dunal

Ar. S. diflorum Vell.

Ar. S. erianthum D.Don

Ab. S. schlechtendalianum Walp.

B. S. verbascifolium Banks ex Dunal

Ar. S. umbellatum Mill.

STAPHYLEACEAE

Am. Turpinia occidentalis (Sw.) G. Don 


\section{THEACEAE}

Am. Cleyera theaeoides (Sw.) Choisy

Ab. Ternstroemia huasteca B.M. Barthol.

\section{TILIACEAE}

Ab. Heliocarpus appendiculatus Turcz.

Ab. H. donnellsmithii Rose

H. Triumfetta acracantha Hochr.

Ar. T. lappula L.

\section{UMBELLIFERAE}

H. Apium leptophyllum (Pers.) F. Muell. ex Benth.

H. Arracacia atropurpurea (Lehm.) Benth. et Hook.

H. Hydrocotyle mexicana Cham et. Schltdl.

H. Sanicula liberta Cham. et Schltdl.

\section{URTICACEAE}

Ar. Myriocarpa longipes Liebm.

H. Pilea pubescens Liebm.

Ar. Urera caracasana (Jacq.) Griseb.

\section{VALERIANACEAE}

B. Valeriana scandens L. var. candolleana (Gardner) C.A. Mull.

H. V. sorbifolia Kunth

\section{VERBENACEAE}

Ar. Lantana hirta Graham

\section{VITACEAE}

B. P. quinquefolia (L.) Planch

B. Vitis popenoei J.H. Fennell 